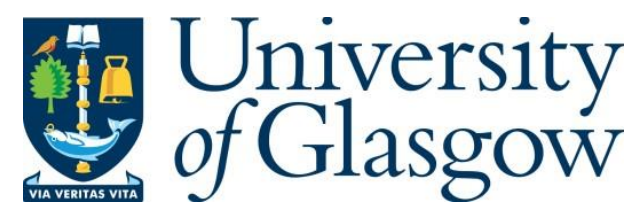

Khamis, M. , Schuster, N., George, C. and Pfeiffer, M. (2019) ElectroCutscenes: Realistic Haptic Feedback in Cutscenes of Virtual Reality Games Using Electric Muscle Stimulation. In: Proceedings of the 25th ACM Symposium on Virtual Reality Software and Technology, Sydney, Australia, 12-15 Nov 2019, p. 13. ISBN 9781450370011 (doi:10.1145/3359996.3364250).

There may be differences between this version and the published version. You are advised to consult the publisher's version if you wish to cite from it.

(C) The Authors 2019. This is the author's version of the work. It is posted here for your personal use. Not for redistribution. The definitive Version of Record was published in Proceedings of the 25th ACM Symposium on Virtual Reality Software and Technology, Sydney, Australia, 12-15 Nov 2019, p. 13. ISBN 9781450370011.

http://eprints.gla.ac.uk/197278/

Deposited on: 24 September 2019

Enlighten - Research publications by members of the University of Glasgow http://eprints.gla.ac.uk 


\section{ElectroCutscenes: Realistic Haptic Feedback in Cutscenes of Virtual Reality Games Using Electric Muscle Stimulation}

\author{
Mohamed Khamis \\ mohamed.khamis@glasgow.ac.uk \\ University of Glasgow \\ Glasgow, UK
}

\author{
Nora Schuster \\ Ceenu George \\ LMU Munich \\ Munich, Germany
}

\author{
Max Pfeiffer \\ maxpfeiffer.hci@gmail.com \\ University of Muünster \\ Muünster, Germany
}

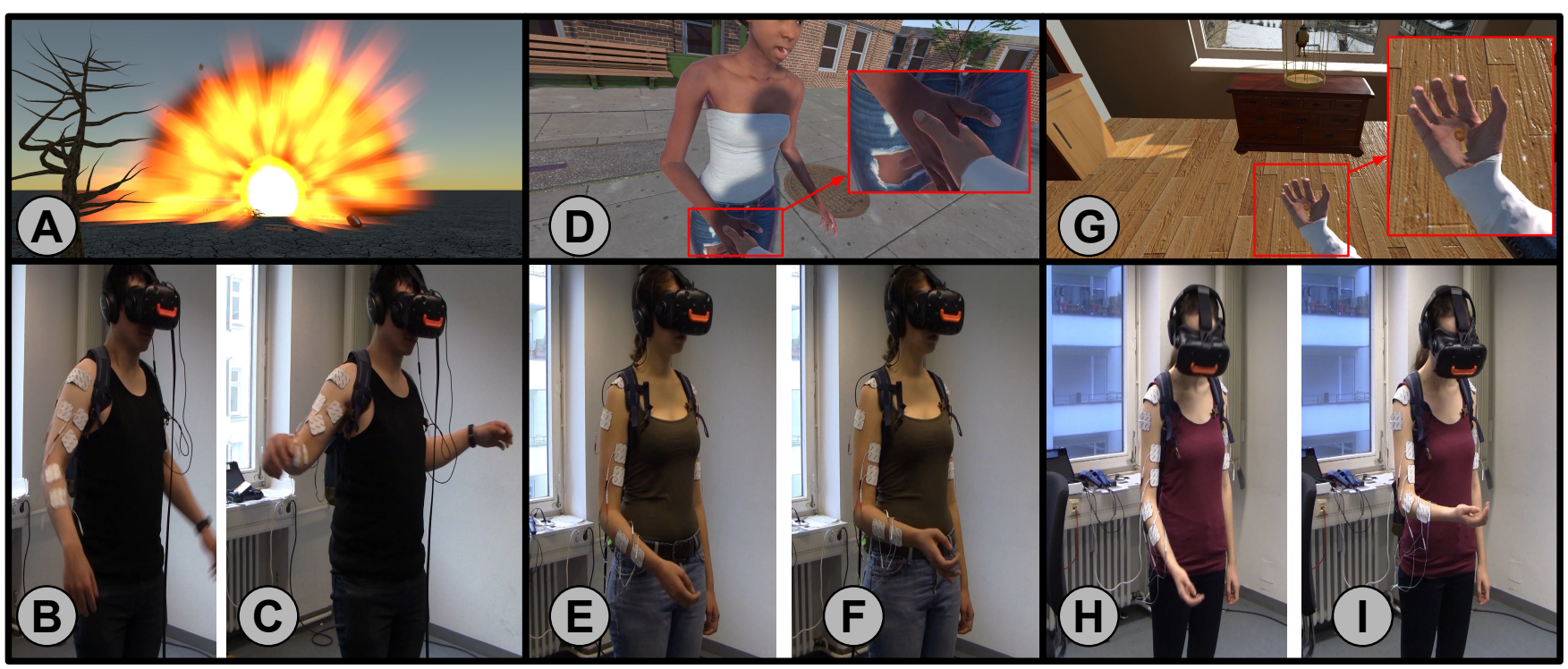

Figure 1: We show that Electric Muscle Stimulation (EMS) significantly increases perceived realism and presence of in-game cinematics in VR games. In (A), the user's arms are inadvertently extended via EMS in an explosion scene. In (D), a handshake is simulated by EMS when greeting a virtual avatar. In (G), the user's forearm is raised via EMS, and hands are opened to find a key to a bird's cage. Although we do not actuate hand rotation via EMS in (I), actuating the forearms and opening the hands via EMS while showing a virtual hand facing upwards nudges users to intuitively rotate their hands.

\begin{abstract}
Cutscenes in Virtual Reality (VR) games enhance story telling by delivering output in the form of visual, auditory, or haptic feedback (e.g., using vibrating handheld controllers). Since they lack interaction in the form of user input, cutscenes would significantly benefit from improved feedback. We introduce the concept and implementation of ElectroCutscenes, where Electric Muscle Stimulation (EMS) is leveraged to elicit physical user movements to different body parts to correspond to those of personal avatars in cutscenes of VR games while the user stays passive. Through a user study $(\mathrm{N}=22)$ in which users passively received kinesthetic feedback resulting in involuntarily movements, we show that ElectroCutscenes significantly increases perceived presence and realism compared to controller-based vibrotactile and no haptic feedback.
\end{abstract}

VRST '19, November 12-15, 2019, Parramatta, NSW, Australia

(C) 2019 Association for Computing Machinery.

This is the author's version of the work. It is posted here for your personal use Not for redistribution. The definitive Version of Record was published in 25 th ACM Symposium on Virtual Reality Software and Technology (VRST '19), November 12-15, 2019, Parramatta, NSW, Australia, https://doi.org/10.1145/3359996.3364250.
Furthermore, we found preliminary evidence that combining visual and EMS feedback can evoke movements that are not actuated by either of them alone. We discuss how to enhance realism and presence of cutscenes in VR games even when EMS can partially rather than completely actuate the desired body movements.

\section{CCS CONCEPTS}

- Human-centered computing $\rightarrow$ Virtual reality; Haptic devices; • Software and its engineering $\rightarrow$ Interactive games.

\section{KEYWORDS}

EMS, Haptic Feedback, Haptics, Head-mounted Displays

\section{ACM Reference Format:}

Mohamed Khamis, Nora Schuster, Ceenu George, and Max Pfeiffer. 2019. ElectroCutscenes: Realistic Haptic Feedback in Cutscenes of Virtual Reality Games Using Electric Muscle Stimulation. In 25th ACM Symposium on Virtual Reality Software and Technology (VRST '19), November 12-15, 2019, Parramatta, NSW, Australia. ACM, New York, NY, USA, 10 pages. https: //doi.org/10.1145/3359996.3364250 


\section{INTRODUCTION}

Despite the myriad of applications of virtual reality (VR), VR games pertain as one of the most prevalent uses of VR. Games, including VR games, are almost always augmented with cutscenes that enhance the story telling, immerse players, or introduce the players to rules, characters and hints in the game. Cutscenes are in-game cinematics that are usually non-interactive, but rather leverage the system's output modalities to enhance the gaming experience for a passive rather than an active user. Cutscenes have been argued to be very important to a game's success [21].

Cutscenes have been limited mainly to visual, auditory and simple tactile feedback. While research brought forth a plethora of novel haptic feedback concepts for VR [11, 20, 24, 26], haptic feedback for non-interactive experiences in immersive environments, such as VR game cutscenes and 360 degree videos, is underinvestigated. Cutscenes are a) shorter and can benefit from focused immersive experiences, and b) non-interactive, thus less immersive by design, which means they can benefit from improved immersion.

We propose ElectroCutscenes, a concept that leverages Electric Muscle Stimulation (EMS) to deliver highly immersive and realistic cutscenes in VR games that elicit high subjective presence. This is done by actuating body parts in sync with events that are happening in the game's cutscene. This results in passive users receiving kinesthetic (forced) feedback thereby moving their limbs involuntarily. For example, as the player's virtual avatar raises its forearm and opens its hand to find a key (Figure 1G), the player's forearm is raised, and their fingers are extended via EMS (Figure 1H-I). This means that our approach flips typical gaming mechanics where players control their avatar's movements, and instead reflects the movements of the virtual avatar onto the player's body. This sets this work apart from previous work in EMS-based haptic feedback in VR [37, 38, 40], since we look into reflecting the avatar's movements on the player's body in non-interactive scenarios, i.e., users are passive during cutscenes and receive the system's output rather than providing input themselves.

A further difference to previous work is that we investigate how EMS feedback can still increase the immersion of users who are "passive viewers" of VR cutscenes. This allowed us to uncover preliminary evidence for novel aspects in the interplay between haptic feedback and immersive virtual environments. For example, in Figure 1G-I, we actuate the participant's forearm and extend her fingers, but we do not rotate her hand to face upwards. Nevertheless, participants of our study rotated their hands upwards in response to seeing their virtual hand facing upwards. This was only observed when using EMS, and not when perceiving visual feedback only or handheld controller based vibtrotactile feedback (we will refer to it from now on as "Vibrotactile Feedback" for short). This means that in addition to increasing realism and presence in VR cutscenes, our findings suggest that it might not be necessary to actuate the entire body movement using EMS; instead, EMS can be combined with visual feedback to nudge participants to subconsciously complete missing movements that were not actuated. That is, users tend to "complement EMS actuations" when the movement is triggered by EMS and supported by visual feedback. This presents an important development in the use of EMS for HCI applications, because it is sometimes challenging to actuate certain body parts due the muscles being deep, being small, or requiring long calibration times.

This work contributes 1) the concept and implementation of ElectroCutscenes, and 2) an understanding of the impact of using EMS-based haptic feedback in cutscenes of VR games based on findings from a user study $(\mathrm{N}=22)$ in which we compared ElectroCutscenes to two baselines: no-feedback, and vibration-based feedback delivered through handheld controllers.

\section{RELATED WORK}

Our work builds on two strands of previous work: (1) the use of electric muscle stimulation (EMS) in HCI, and (2) haptic feedback in virtual reality $(\mathrm{VR})$.

\subsection{EMS in $\mathrm{HCI}$}

While Electric Muscle Stimulation was traditionally used for therapeutic purposes [58], EMS has recently attracted the attention of HCI researchers as a novel mean for haptic feedback. Lopes and Baudisch argue that EMS can be leveraged to "pack strong mechanical actuations into [tiny wearable devices]" [36], rather than building larger human actuation devices such as exoskeletons. For example, it has been used in PossessedHand [55] to stimulate forearm muscles to perform complex tasks, such as playing musical instruments. EMS was used to assist in playing [42] and also learning how to play musical instruments [13]. In Affordance++, Lopes et al. leveraged EMS to communicate the dynamic use of every day objects, e.g., if a cup is too hot to touch, the user's arm would be actuated to move away from the cup [39].

Other researchers moved away from actuating arm muscles to full body actuations. For example, Hassib et al. exploited EMS to remotely communicate emotional responses to other parties by actuating different parts of the body (e.g., extending arms in case of happiness) [23]. Hassan et al. implemented FootStriker [22], which leveraged EMS in footwear to protect from knee-related injuries by adjusting the foot's pose while running. Pfeiffer et al. proposed cruise control for pedestrians to steer users via EMS as they walk [45]. Goto et al. actuated parts of the face to enforce smiling [16]. Niijima and Ogawa used EMS on the masseter muscle near the jaw to communicate virtual food textures [43]. Lopes et al. used EMS to enable proprioceptive interaction by, for example, actuating limbs based on the progress of a video [40].

\subsection{Haptic Feedback in VR}

Haptic feedback has been of particular interest for games, and was adopted as early as 1997 in gaming consoles such as Nintendo 64 and Sony Playstation [3]. Today's commercial Head-Mounted Displays (HMDs), such as HTC Vive and Oculus Rift, come with controllers equipped with vibration motors to provide tactile feedback in the form of vibration.

Researchers explored novel means for delivering haptic feedback in VR. Recent examples include Haptic Links [24], in which handheld controllers were augmented with electro-mechanical connectors to allow bimanual haptics. CLAW enhanced haptic feelings of grasping, touching and triggering in VR [9]. In Sparse Haptic Proxy, eye tracking is used to predict where users will touch a surface in VR, and then the user's hand is redirected to touch a physical 
proxy that provide tactile sensations [7]. Researchers employed robot arms [2], airflow [19, 53], water streams [25], exoskeletons [18], and pneumatics [10] for haptic feedback. Tactile feedback was explored at different parts of the body, such as at the feet [41, 48, 49], back [26], fingertips [50], hands [54], and arm [37]. Some works looked into leveraging human workers to provide haptic feedback; "Turkers" manually lifted, tilted, and pushed the user's limbs or torso for haptic feedback [5], or placed walls and doors around the user to produce haptic sensations [8]. In Mutual Human Actuation, players in different VR games provide haptic feedback to one another [6]. Other researchers used drones to provide haptic feedback. Knierim et al. used mini-quadcopters equipped with tips that hit different user body parts to, for example, simulate a bee sting [30]. Abdullah et al. used a Drone to simulate weight bearing in VR [1]

\subsection{EMS for Haptic Feedback in VR}

We are not the first to use EMS for haptic feedback in VR games $[14,34]$. Jain et al. employed EMS to augment light saber duels in a VR game [27]. Parts of the face where actuated by Koto et al. [32] to extend VR experience with illusions of fear and pain. Lopes et al. studied the design of haptic feedback in VR [40]. They identified hard design, where users receive continuous EMS feedback to continuously perceive a VR object, soft design, where users can slightly penetrate VR objects, and repulsion design, where hands are propelled away from the object. Impacto [37] is an example of repulsion design, where EMS renders haptic sensation of being hit (e.g., punched) in VR games. More recently, Lopes et al. introduced a mobile system that enhances mixed reality experiences using EMS, by actuating the user's muscles while the user is interacting with physical objects [38]. Mainly, the user is actively involved in those interactions with the VR scenes and receives EMS responses based on that. Some VR Companies, such as TeslaSuit [33], sell suits with electrodes that actuate different body muscles.

\subsection{Comparison to Previous Work}

Despite the myriad of work on EMS in interactive VR (e.g., EMS in VR games [37]), it remains unclear how the experience of passive users who are not interacting with the virtual scene can be improved via EMS. Compared to previous work, a core difference in our work is that we investigate VR game cutscenes, i.e., non-interactive experiences in VR, where the user perceives cinematics. This makes our work different from work on using EMS in interactive VR applications in that the user is passively perceiving kinesthetic feedback rather than voluntarily reacting to the environment. The only exception is the work of Kono et al. [31] who studied inducing fear and pain while the user is passive in VR. Our work is different than theirs in that we explore kinesthetic feedback to induce movements rather than inducing emotions. These differences allowed us to 1) significantly improve the immersiveness of cutscenes, which are otherwise less immersive due to lack of user involvement, and 2) uncover initial evidence of novel effects of combining EMS and visual feedback.

\section{CONCEPT AND DESIGN}

ElectroCutscenes improves realism by actuating the user's body via EMS to mirror the virtual avatar's movements in VR cutscenes. It is

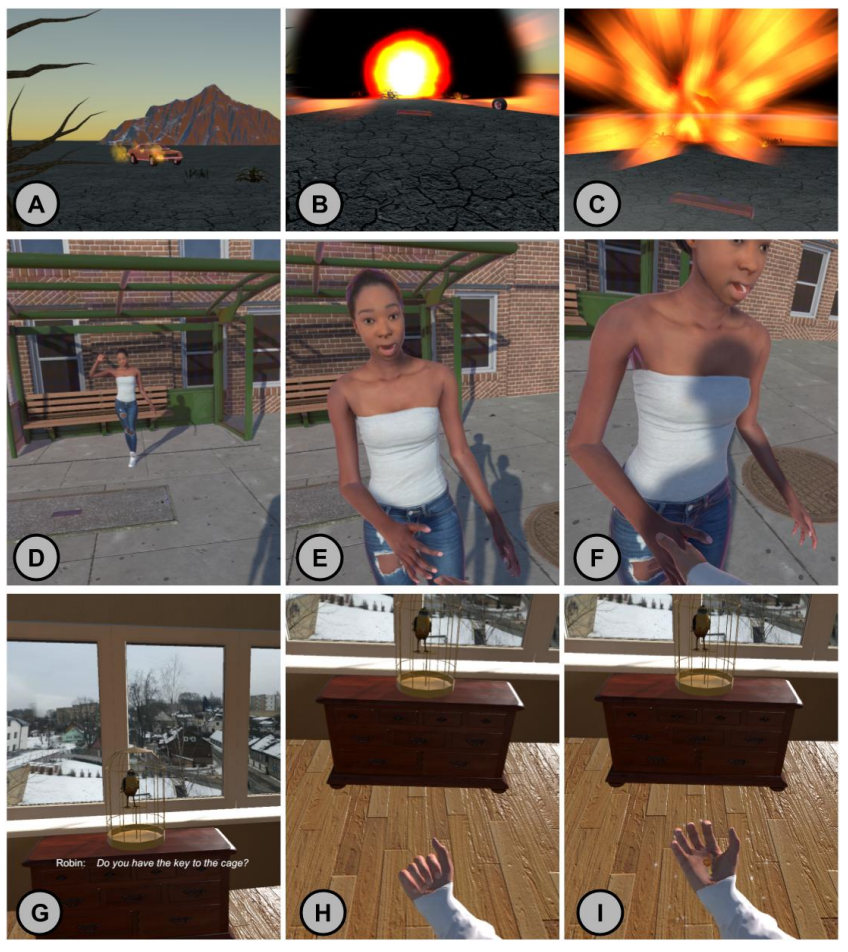

Figure 2: In the first scene (A-C) a car approaches the user, it explodes and sends shock waves towards her. In the second (D-F), an avatar waves to the user, then approaches and gives its hand for a handshake. In the last scene (G-I), a bird asks the user for a key. The user's arm is raised, and hands are opened to show a key. In all scenes, we actuated the user's real body using EMS to mirror the avatar's movements. composed of 3 components: 1) the cutscenes, 2) muscle actuation, and 3) EMS feedback design.

\subsection{Cutscenes}

Cutscenes are in-game cinematics that are displayed during gameplay. They are often not interactive, but instead they deliver certain content to the user with the aim of enhancing story telling [21]. Games often show cutscenes to set the scene, introduce game characters, give hints to the player, or show consequences of the player's actions. Feedback in cutscenes has been mostly limited to visual, auditory, and simple tactile feedback delivered through vibrating handheld controllers - other feedback modalities for cutscenes are relatively under investigated. Our aim is to deliver more realistic haptic feedback, where the movements of the player's avatar are reflected onto the user's physical posture.

In the following, we introduce the cutscenes that we extend with haptic feedback. We then explain how vibrotactile feedback is typically provided in those scenes, and how feedback using electric muscle stimulation (EMS) can be leveraged in them to enhance perceived realism and presence.

3.1.1 Cutscene 1: Explosion. In-game explosions are widely used in many gaming genres, and are particularly important to the entertainment industry $[15,57]$. This is reflected by the abundance of work about rendering explosions in games [15, 28, 52, 57]. 
As illustrated in Figure 2A-2C, a burning car approaches the user in this scene. The burning car then stops, and then explodes (B) before sending shock waves towards the user (C). Typically, vibrotactile feedback is provided in explosion scenes by a continuous vibration at a frequency relative to the intensity of the explosion for example, a low intensity vibration once the explosion happens, and a more intense one once the shock wave reaches the player Our aim is to deliver realistic haptic feedback via EMS by raising the player's arms and forearms once the shock waves reach her.

3.1.2 Cutscene 2: Handshake. Many in-game cutscenes feature social interactions among game characters. Apart from VR games, there is an increasing trend for social interactions in VR [47].

In the second cutscene (Figure 2D-2F), a virtual avatar greets the user from a distance (D), approaches (E), and then extends her hand to shake the user's virtual hand (F). In such scenes, vibrotactile feedback is often provided by vibrations while hands are shook. Our aim is to provide haptic feedback that moves the user's hand via EMS to simulate a handshake, i.e., raise forearm, grip, and shake forearm vertically. Note that in our realization of this cutscene, the user remains passive and does not move any body parts. Instead, EMS actuation moves the body parts as described above. This means that while the scene seems to be interactive, the user is actually passive.

3.1.3 Cutscene 3: Opening Hands. Item collection is one of the main elements of gaming [17, 35]. Players often have to find and collect tools and items to progress in the game. Cutscenes are often used to instruct the player how to use collected items. For example, an instruction could be: "use the red key to open the red door".

In this cutscene (Figure 2G-2I), a bird asks the player if they have a key for opening the cage $(\mathrm{G})$. The avatar raises its hand $(\mathrm{H})$, then opens it to find the key (I). Vibrotactile feedback can be used in such scenes by delivering vibrations once the key has been found. To enhance immersion and presence in this scene via EMS, the user's forearm should be raised, before rotating and opening the hand. Similar to the previous cutscene, in our realization of this cutscene we asked participants to remain passive and to not move any of their body parts. Instead, the aim is that the EMS feedback actuates the body parts as described above. This means that while the scene seems to be interactive, the user is actually passive.

\subsection{Muscle Actuation and Electrodes Placement}

We first identified the muscles responsible for each relevant movement described in the previous section. The Explosion scene requires raising arms sideways, and raising the forearms. The Handshake scene requires raising the right forearm, performing a grip gesture by flexing the index, middle, ring and little fingers slightly, then finally raise and lower the arm to perform the "shake" of the handshake. The Opening Hands scene requires raising the forearm, rotating the hand, and then extending the fingers. We identified four muscles that need to be actuated to provide the feedback for our three cutscenes. Our choices of muscles and how to actuate them were inspired by prior work on actuating upper body muscles [44] and pilot tests in our lab.

3.2.1 Raising Arms Sideways. The lateral part of the Deltoid muscle is responsible for raising the arms side ways up to a 90 degrees

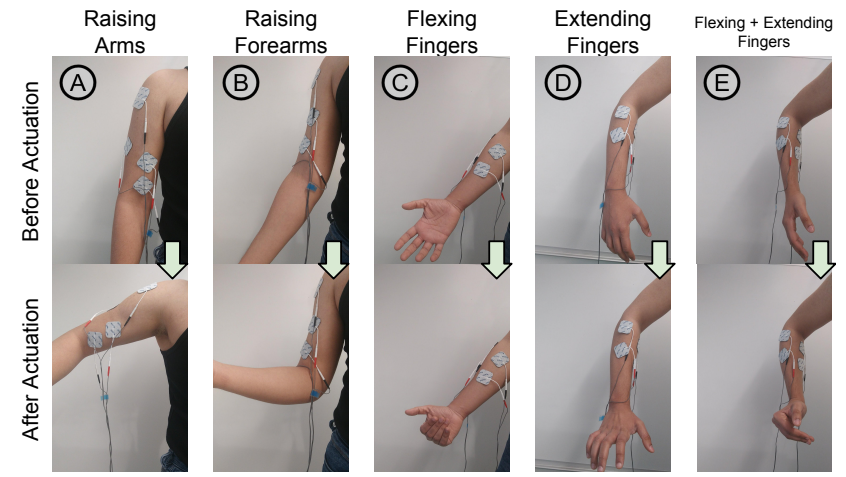

Figure 3: We actuated four different muscles in our scenes. The Deltoid (shoulder) muscle raises the arm side ways until it is at approximately 90 degrees angle (A). The Biceps raise the forearm upwards (B). Flexor digitorum superficialis flexes the four fingers (C). Extensor digitorum extends the same four fingers (D). Actuating the latter two results in a handshake-like gesture (E).

angle. Thus, we actuated this muscle in the Explosion scene for the effect shown in Figure 3A. Since the scene requires both arms, electrodes were placed on the lateral part of both Deltoid muscles.

3.2.2 Raising Forearms. To raise the forearm upwards towards the upper arm, we actuated the Biceps, which lie on the upper arm, between the shoulder and the elbow. Since all three scenes required raising the forearm, we actuated the Biceps in all three scenes for the effect shown in Figure 3B. The Explosion scene requires both arms, so we placed electrodes on the biceps of both arms.

3.2.3 Forearm rotation. Initially we planned to actuate the supinator muscle in the forearm, which is responsible for outwards rotation of the forearm so that the hand's palm would face upwards in the Opening Hands scene. However pilot tests showed that after raising the forearm to the correct position via EMS, participants intuitively rotate their hand at the sight of a virtual arm in this posture. To study this further, we refrained from actuating this muscle during the main study to see if this effect is shared among users. Indeed, 12 out of 22 main study participants rotated their arms intuitively in response to seeing a virtual arm rotating - we discuss this in details in the section 4.5 .

3.2.4 Fingers Flexion, Extension, and Grasp. To flex the four fingers (index, middle, ring, and little fingers), we actuated the flexor digitorum superficialis which lies on the anterior part of the forearm (facing the body), between the elbow and wrist. The muscle's function is to flex the bottom-most joints of all fingers except the thumb. To create an opposite movement, the same four fingers are extended by actuating the extensor digitorum, which lies on the posterior part of the forearm (facing outwards), between the elbow and wrist. For both flexion and extension, electrodes were placed on the right arm's muscle only, the effects are shown in figures $3 \mathrm{C}$ and $3 \mathrm{D}$ respectively. Both muscles were actuated together to create a grasp gesture to be used in the Handshake scene (Figure 3E), while the latter muscle was actuated solely in the Opening Hands scene. 


\subsection{EMS Feedback Design}

In the Explosion scene (Figure 1A-C), we actuated the shoulder first to raise the arms. This was followed by actuation of the biceps to extend the forearms. This required 8 electrodes: 2 on each deltoid and biceps of both arms.

The Handshake scene (Figure 1D-F) started by actuating the biceps to raise the forearm. We then actuated the muscles for flexing and extending the fingers to simulate a handshake. Finally, actuation pulses were used to move the forearm vertically in both directions to simulate a "shake". This required 6 electrodes: 2 on the right biceps, 2 on the right flexor digitorum superficialis, and 2 on the right extensor digitorum.

In the Opening Hands scene (Figure 1G-I), we first actuated the biceps to raise the forearm, before the fingers were extended by another actuation. Again, we left out to rotation movement (which could be done by the supinator muscle) on purpose to see if users realize it and if they perform the missing movement subconsciously. This scene required 4 electrodes: 2 on the right biceps, and 2 on the right extensor digitorum.

\subsection{Implementation}

Our prototype is based on the open source EMS toolkit by Pfeiffer et al. [44]. It consists of (1) an EMS generator, (2) an EMS control module, (3) a desktop PC, and (4) a VR headset.

3.4.1 EMS Configuration. The EMS generator delivers the pulses of electricity to stimulate and hence actuate muscles. Each two output channels of the device are connected to one EMS control module of the toolkit (see Figure 4). We used the STIM-PRO X9+ [56], which features 4 channels. This allows us to activate up to 8 electrodes in parallel, which is sufficient for all scenes. Several parameters have to be set in the EMS generator, including the pulse width, the pulse frequency, and the intensity which reflects the used current strength. The pulse width and frequency are fixed at a $100 \mu \mathrm{s}$ and $100 \mathrm{~Hz}$ in our implementation based on pilot tests and prior work [44]. While the intensity is set during a per-user calibration phase because different intensity levels are required for each user, muscle, position of the electrodes and desired effect $[39,44,45]$. This includes voltage and ampere, which were adjusted in each calibration phase, but were between $0-50 \mathrm{~V}$ and $0-100$ $\mathrm{mA}$.

3.4.2 Triggering EMS Feedback. The EMS control module connects the electrodes and the EMS generator, and decides whether or not to pass the signal to the user or directly back to the device. We use two Arduino-based control modules of the EMS toolkit in our prototype. Each module has two input and two output slots; two cables connected the input slots of each module to two of the EMS generator's channels, thus utilizing all 4 channels (see Figure 4 Haptic enhancement). The modules can be communicated with using BLE. In our final implementation, the commands are sent directly from any platform with Bluetooth 4.0 or higher, such as a PC running Unity. This allows for cutscenes to directly send actuation commands to the EMS control module. However, during the user study it was important to keep the experimenter in the loop, and enable her to abort the actuation in case of any unexpected events. Thus, we implemented an Android application that runs on a mobile device, and can send commands to the modules via Bluetooth during the experiment (see Figure 4). The experimenter can use this app to activate actuations (e.g., start scene A's actuation sequence) or abort them in case of an emergency. The control module is powered by 9 volt batteries.

We used a desktop PC with Intel Core i7 6500k processor, an NVidia GTX 1080 graphics adapter, and 16 GB RAM running Windows 10 . The system ran Unity VR, which displayed the scenes on the HTC Vive VR headset.

\section{EVALUATION}

The goal of this user study is to evaluate the effectiveness of our approach in enhancing presence and realism during cutscenes in virtual reality games. To do so, we invited 22 participants (14 females) to experience the EMS feedback for the three cutscenes we described. Participants were aged between 19 and 30 years $(M=24$, $S D=3$ ). We added two baseline conditions; participants watched the scenes without any feedback, and watched them while carrying handheld controllers that deliver vibration-based feedback as done in current commercial systems.

\subsection{Apparatus}

The study took place in our lab. As shown in Figure 4, participants wore an HTC Vive VR headset as well as headphones. In the vibration condition, they additionally held an HTC Vive controller in each hand. As explained previously, it was important to allow the experimenter to abort the EMS signal in case of an emergency. Therefore the experimenter was provided an Android phone with an app capable of 1) activating the actuation sequence for the whole scene with a single button press at the beginning of the scene, and 2) aborting the EMS signals at any time. On the other hand, since adverse effects of vibrotactile feedback are less likely, vibrations of the handheld controllers were triggered through Unity in the manner explained earlier.

We attached 12 electrode pads to both arms of the participants: 2 electrodes $\times$ Biceps on both arms + Deltoid on both arms + flexor digitorum superficialis on right arm + extensor digitorum on right arm. We used a pair of $5 \mathrm{~cm} \times 5 \mathrm{~cm}$ electrode pads for each muscle except for the deltoid (shoulder) muscle, for which one of the pairs was a larger $10 \mathrm{~cm} \times 5 \mathrm{~cm}$ pad. The EMS generator and the control modules were placed in a backpack that the participants put on.

\subsection{Study Design and Procedure}

We first welcomed the participants, introduced EMS and VR, explained the study, and asked them to sign a consent form and fill in a demographics questionnaire. Participants went through the calibration phase that we describe in details in the next section. They were then equipped with the HTC Vive and headphones, both of which were connected to a PC running the VR scenes within Unity. In the vibration condition, participants were also asked to hold the HTC Vive controllers. They were asked to keep their arms relaxed, to only look forward and stay at the starting position.

The study was designed as a repeated measures experiment with one independent variable being the feedback modality. The independent variable had three conditions: 1) EMS feedback, 2) 


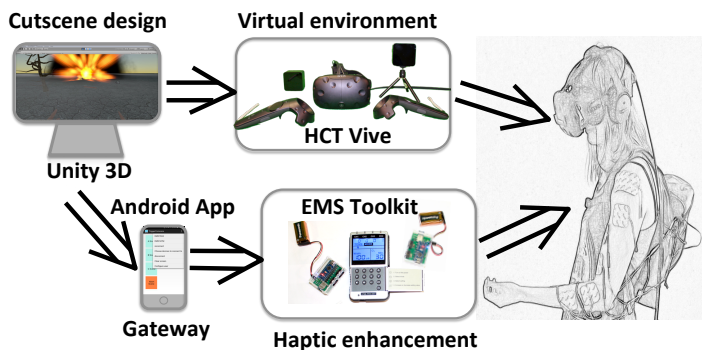

Figure 4: Participants were equipped with 12 electrodes targeting the muscles needed for the scenes, and put on a HTC Vive headset in addition to headphones. In the vibration condition, participants held HTC's handheld controllers to deliver the vibration feedback. The experimenter in the loop during the experiment, she used an Android app to trigger the actuations required for every scene, and to stop the muscle stimulation to abort if needed.

(Controller-based) Vibration feedback, and 3) No Feedback. Participants went through three blocks - one per condition - in which they watched all three cutscenes. The order of blocks and scenes was counter balanced across participants using a Latin-square.

After each block, participants were asked to rate their perceived presence by filling the IGroup Presence Questionnaire (IPQ) [51] and a customized questionnaire inspired by previously work on EMS in VR [37, 40] in which we asked about perceived realism and consistency of the feedback with the scene (7-points Likert scale). We video recorded all sessions with an RGB camera for later analysis. After all trials, participants ranked the feedback methods, and underwent a semi-structured interview.

4.2.1 Choice of Vibration Controller Placement. We decided to let participants hold the HTC Vive controllers, which is state of the art in commercial systems. An alternative design could have been to place vibration motors directly on the same positions where we have put the EMS electrodes [46]. However, such a design would have results in an unfair disadvantage to the (Controller-based) Vibration feedback. For example, EMS actuates a user's forearm and hands although the electrodes are on the biceps (e.g., see Figure 3). Thus, mimicking the feedback using vibration motors should be designed in a way such that the hands and not the biceps are affected. For these reasons, we decided that participants of our study would hold a vibration controller in each hand.

\subsection{Calibration Phase}

Participants underwent a calibration session before going through any condition, during which the parameters of the EMS generator were determined for each participant. During the calibration participants had their arms relaxed first and then we performed the stimulation again with the arm raised, like it would be during the experiment later. Movements that required multiple muscle actuations were also checked. Both arms were calibrated independently. We gradually increased the EMS intensity in the calibration phase to achieve the desired results. We ensured that there were clear visually perceivable involuntary movements in each cutscene in

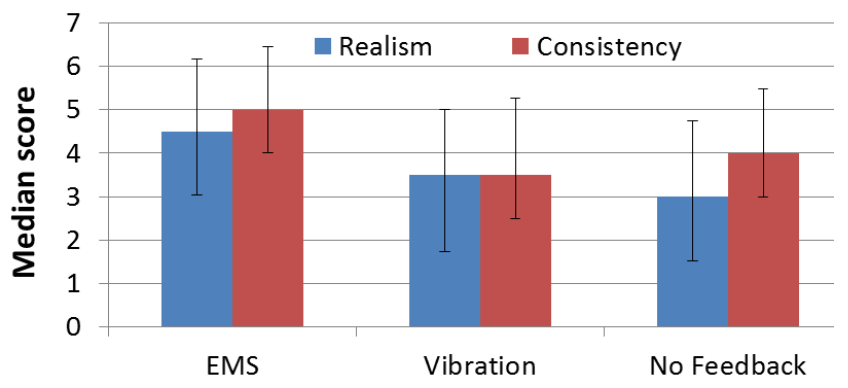

Figure 5: When asked on a 7-point Likert scale ( $7=$ strongly agree; $1=$ strongly disagree) on the realism of the feedback, and its consistency with the scene, participants indicated that EMS feedback is the most realistic, and the most consistent with the scene. The error bars indicate the standard deviation.

the way we expected, i.e., the arms moves clearly up and down during the hand shake scene. We ensured that the stimulation was comfortable and not painful. No participants reported pain during the study. As recommended in prior work [44], the EMS intensity was set to be strong enough to enforce passive movements, but weak enough to allow users to override it. Participants were told they could override the signal, and some tried to do that in the calibration phase. The process took 10 to 50 minutes per participant. We did not want participants to put on and take off the pads during the experiment lest it influences their perception of any of the modalities. Hence we left the pads on the participant, but disconnected them when unneeded.

\subsection{Limitations}

We used 3 cutscenes for each of the feedback methods. While we do not claim that the quantitative results will remain the same if the study is replicated for other cutscenes, we expect the relative results to be the same. We focused on comparing ElectroCutscenes with vibrotactile feedback since it is common on today's systems. But unlike tactile feedback, ElectroCutscenes generates kinesthetic movements. This means that results that are in favor of ElectroCutscenes could also be valid for technologies that generate kinesthetic movements (e.g., exoskeletons [18]). We also acknowledge that the effect of the vibration controller might be attributed not only to the vibration feedback, but also to the fact that the user is holding a controller.

EMS technology is limited in that it requires 1) electrodes placement and 2) time-consuming calibration [39, 44, 45]. Recent research proposed automatic calibration using EMS-arrays or "sleeves" $[12,16,29,33]$. Therefore we envision that developers will have access to a wide range of muscles that they could program cutscenes to actuate - the TeslaSuit [33] is an evidence that we are moving towards this vision. This will make it possible to apply EMS to many cutscenes without altering the arrangement of electrodes. Advances in this direction will make the concept of ElectroCutscene straightforward to realize in the near future.

Finally, EMS-based actuations come with risk as the user's view of the real world is blocked by the HMD; involuntary movements may result in injury to the user or bystanders. For example, the user 


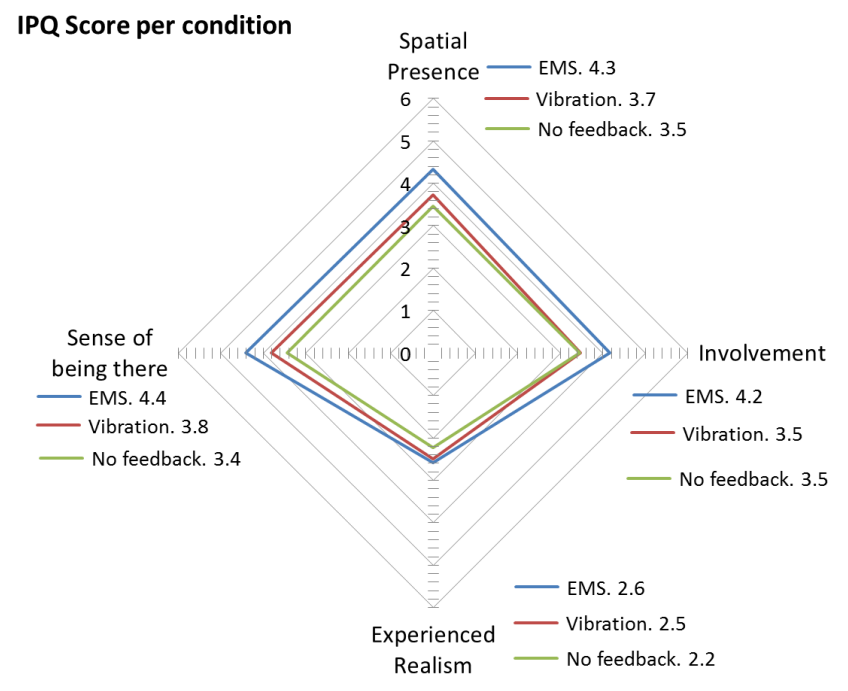

Figure 6: Mean IPQ scores indicate that EMS results in higher presence, sense of being there, involvement, and realism. The IPQ Score relates to the sense of presence experienced in a virtual environment. $6=$ highest IPQ Score (feeling present); 0 = lowest IPQ Score (feeling not present).

might inadvertently thrust their arms into a sharp object or a person. This can be easily counteracted by, for example, detecting when bystanders are near the user and disable actuations accordingly. Alternatively, the user can be guided to a position in the room where it is safe to perform arm movements.

\subsection{Results}

4.5.1 Realism and Consistency with the Scene. As Figure 5 illustrates, participants found EMS feedback to result in more realistic experiences, and to be more consistent with the scene compared to vibration-based feedback and no feedback. A non-parametric Friedman's test revealed a significant effect of the feedback method on both the perceived realism $\left(\chi^{2}(2)=9.0, p<0.05\right)$ and consistency $\left(\chi^{2}(2)=6.177, p<0.05\right)$ with the scene depending on the feedback method. Post hoc pairwise comparisons using Wilcoxon signed-rank test with Bonferroni correction showed that EMS feedback $(M d n=4.5, S D=1.67)$ results in statistically significantly higher perceived realism, compared to no feedback $(M d n=3$, $S D=1.73)(p<0.05)$. In terms of consistency with the scene, a Wilcoxon signed-rank test with Bonferroni correction also showed that EMS feedback $(M d n=5, S D=1.46)$ results in statistically significantly higher consistency compared to vibration $(M d n=3.5$, $S D=1.76),(p<0.05)$, and compared to no feedback $(M d n=4$, $S D=1.48),(p<0.05)$. The other pairs were not statistically significant $(p>0.05)$.

This means that EMS results in the highest perceived realism and consistency with the scene.

4.5.2 Preference. When asked to rank the feedback methods according to their preference, 14 out of 22 preferred EMS the most, while 7 preferred vibration, and only one preferred no feedback. A weighted ranking score indicates that EMS (score $=54$ ) is the most preferred feedback technique, followed by vibration (score $=45$ ), and no feedback (score=33).

4.5.3 Presence Questionnaire. Figure 6 illustrates the results from IGroup's standardized questionnaire (IPQ), which features subscales for 1) spatial presence, 2) involvement, 3) realism, 4) sense of being there. We followed the methodology by Schubert et al. to analyze and interpret the results [51]. The results show that EMS feedback outperforms the two other conditions on all subscales. EMS elicited the highest spatial presence $(M=4.3, S D=0.85)$ compared to vibration $(M=3.7, S D=1.01)$ and no feedback $(M=3.5, S D=$ 1.17). EMS was also associated with a stronger involvement $(M=$ $4.2, S D=1.24)$ compared to vibration $(M=3.5, S D=1.09)$ and no feedback $(M=3.5, S D=1.25)$. Experienced realism was reported to be higher with EMS $(M=2.6, S D=1.32)$ compared to vibration $(M=2.5, S D=1.27)$ and no feedback $(M=2.2, S D=1.10)$. Finally, the sense of being there was highest with EMS $(M=4.4$, $S D=1.4)$, followed by vibration $(M=3.8, S D=1.6)$, and no feedback $(M=3.4, S D=1.35)$.

To check for statistical significance, we ran repeated measures ANOVA tests with Greehouse-Geisser correction with the assumption of sphericity was violated. We found a significant main effect of feedback modality on spatial presence $F_{1.86,16.8}=7.73, p<0.005$, involvement $F_{1.96,41.11}=7.18, p<0.005$, and sense of being there $F_{1.9,39.97}=3.97, p<0.05$. Post hoc analysis with Bonferroni correction showed that EMS feedback elicited significantly higher sensed presence compared to vibration feedback $(p<0.01)$ and no feedback $(p<0.01)$. EMS feedback also results in significantly higher sense of presence compared to no feedback $(p<0.001)$ and vibration feedback $(p<0.05)$.

This means that EMS feedback outperforms vibrotactile and no feedback according to the IPQ questionnaire.

4.5.4 Observations. We analyzed video recordings of the study to find key observations. Although we did not apply the necessary electrodes for rotating the arm upwards in the Opening Hands scene, none of the participants reported that they felt any movements were missing. In fact, 12 out of 22 participants rotated their hand to face upwards in response to seeing their virtual hand facing upwards (see Figure 7), and at the same time did not report performing any movements consciously. This effect was observed only in the EMS condition, but not in the no-feedback or vibration condition. This suggests that combining EMS with visual feedback can nudge participants to subconsciously complement the EMS actuations and perform movements that would have not been inadvertently performed using EMS alone, or visual feedback alone.

4.5.5 Interviews. Participants found that EMS feedback "very realistic" $(\mathrm{N}=11)$ and makes the experience "positively intense" $(\mathrm{N}=3)$, and made them "more involved in the explosion yet safe" P15. P5 praised that their body is reacting without them doing anything. P17 particularly liked that the feedback is not only addressing her hands. P21 admired that the EMS feedback mirrors what she felt like doing. On the downside, P7 and P10 felt the actuations are not $100 \%$ identical to the virtual avatar's movements. P22 was very negative towards both EMS and vibration feedback. She found EMS unnatural and strange, and did not like that she has to hold a vibrating controller. Three participants stated that although they liked 

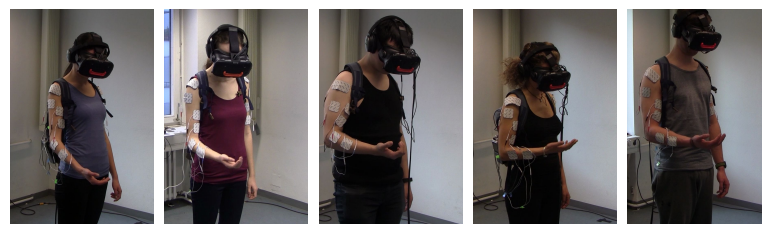

Figure 7: Although only the biceps and not the muscle required for rotating hands to face upwards were actuated, participants rotated their hands when their virtual faced upwards. This suggests that EMS-based feedback must not necessarily actuate the entire movement. Instead, EMS can partially actuate movements, while visual feedback nudges users to subconsciously complete the entire movement.

EMS feedback, they felt that they were able to pay more attention to the visual and auditory details in the no feedback condition.

\section{DISCUSSION}

ElectroCutscenes was found to significantly improve perceived realism, presence and involvement in cutscenes of VR games by actuating the user while they passively perceive the scenes. We also found that although some movements could be challenging to actuate via EMS, users might be intrigued to "complement" the actuations and perform the remainder of the movement themselves. Through developments such as TeslaSuit, EMS sleeves and arrays $[12,16,29,33]$, we envision that developers of cutscenes will be able to program them to evoke certain movements on the player's body, such as hands, arms, legs, stomach and back.

\subsection{Complementing EMS Actuations}

A challenge in $\mathrm{HCI}$ research that utilizes EMS is that some muscles are very difficult to stimulate, either because they are too small or deep inside the body. Our findings provide initial evidence that it might not be necessary to be able actuate each and very muscle of the human body. Instead, combining other simpler modalities along with EMS could trigger users to complement the actuations by performing the entire expected movement. This is however a sidefinding in our study which we merely consider as a preliminary result that needs to be confirmed in a future research. Further research is needed to understand which types of movements can benefit from this phenomena, and to identify best practices for nudging users to drive them to complete the movement themselves.

\subsection{Non-voluntary and Voluntary Movements}

Voluntarily movements such as handshakes require users to consciously move parts of their body. Here EMS can only complement the natural movements that users perform, but can also distort the regular perception-cognition-action loop, possibly resulting in less presence and realism. On the other hand, involuntary actions such as reactions to explosions, or to touching hot objects, do not normally occur in VR. Here EMS does not only provide haptic feedback, but it also results in movements that would not otherwise be performed by participants. Hence the use of EMS for involuntary movements, which are common in cutscenes, is more promising for improving immersion. Further work is needed to understand the impact of EMS in voluntary vs non-voluntary actions.

\subsection{Applicability to $360^{\circ}$ Videos}

$360^{\circ}$ videos are gaining popularity with the increased performance and affordability of high quality HMDs [4]. While we focused on shorter and more focused cutscenes in VR games, many of the aspects of this work are also applicable to $360^{\circ}$ videos. A challenge in $360^{\circ}$ videos however is to accommodate for cases when the user is attending to different directions in the environment. For example, if an explosion happens in front of the user, the haptic EMS-based feedback should be very different than if it occurs behind the user.

\subsection{Future Work}

Our findings open doors for future research in understanding the interplay between EMS and immersive VR. One direction for future work is to develop a design space for aspects that lead users to complement EMS actuations. For example, a better understanding of whether immersion (e.g., how realistic the user's virtual hands are), the user's own experience, enjoyment, or other factors influence the user's tendency to complement EMS actuations.

Future versions of ElectroCutscenes can benefit from integration with motion sensing. For example, if the user's back is facing the explosion, the arms could be actuated differently to simulate shock waves directed at the user's back.

\section{CONCLUSION}

In this work, we introduced the concept and implementation of ElectroCutscenes. ElectroCutscenes delivers highly immersive and realistic cutscenes for VR games by leveraging electric muscle stimulation to actuate users as they passively perceive the scenes. We conducted a user study in which we compared the perception of EMS-based feedback to vibrotactile feedback through handheld controller and no feedback. Results are in favor of EMS, demonstrating that it results in higher perceived realism, involvement, and presence. We discussed opportunities of integrating EMS with visual feedback in immersive virtual environments. We found that actuating body movements partially could result in the users performing the rest of the movement when shown appropriate visual feedback.

\section{REFERENCES}

[1] Muhammad Abdullah, Minji Kim, Waseem Hassan, Yoshihiro Kuroda, and Seokhee Jeon. 2017. HapticDrone: An Encountered-Type Kinesthetic Haptic Interface with Controllable Force Feedback: Initial Example for 1D Haptic Feedback. In Adjunct Publication of the 30th Annual ACM Symposium on User Interface Software and Technology (UIST '17). ACM, New York, NY, USA, 115-117. DOI: http://dx.doi.org/10.1145/3131785.3131821

[2] Bruno Araujo, Ricardo Jota, Varun Perumal, Jia Xian Yao, Karan Singh, and Daniel Wigdor. 2016. Snake Charmer: Physically Enabling Virtual Objects. In Proceedings of the TEI '16: Tenth International Conference on Tangible, Embedded, and Embodied Interaction (TEI '16). ACM, New York, NY, USA, 218-226. DOI : http://dx.doi.org/10.1145/2839462.2839484

[3] Shantanu Bala. 2016. A Brief History of Haptic Feedback in Video Games. https:// blog.somaticlabs.io/a-brief-history-of-haptic-feedback-in-video-games/. (2016). Accessed 10 September 2018.

[4] Samantha W Bindman, Lisa M Castaneda, Mike Scanlon, and Anna Cechony. 2018. Am I a Bunny? The Impact of High and Low Immersion Platforms and ViewersâĂŹ Perceptions of Role on Presence, Narrative Engagement, and Empathy during an Animated $360^{\circ}$ Video. In Proceedings of the 2018 CHI Conference on Human Factors in Computing Systems (CHI '18). ACM, New York, NY, USA, 12.

[5] Lung-Pan Cheng, Patrick Lühne, Pedro Lopes, Christoph Sterz, and Patrick Baudisch. 2014. Haptic Turk: A Motion Platform Based on People. In Proceedings of the 32Nd Annual ACM Conference on Human Factors in Computing Systems 
(CHI '14). ACM, New York, NY, USA, 3463-3472. DOI : http://dx.doi.org/10.1145/ 2556288.2557101

[6] Lung-Pan Cheng, Sebastian Marwecki, and Patrick Baudisch. 2017a. Mutual Human Actuation. In Proceedings of the 30th Annual ACM Symposium on User Interface Software and Technology (UIST '17). ACM, New York, NY, USA, 797-805. DOI : http://dx.doi.org/10.1145/3126594.3126667

[7] Lung-Pan Cheng, Eyal Ofek, Christian Holz, Hrvoje Benko, and Andrew D. Wilson. 2017b. Sparse Haptic Proxy: Touch Feedback in Virtual Environments Using a General Passive Prop. In Proceedings of the 2017 CHI Conference on Human Factors in Computing Systems (CHI '17). ACM, New York, NY, USA, 3718-3728. DOI : http://dx.doi.org/10.1145/3025453.3025753

[8] Lung-Pan Cheng, Thijs Roumen, Hannes Rantzsch, Sven Köhler, Patrick Schmidt, Robert Kovacs, Johannes Jasper, Jonas Kemper, and Patrick Baudisch. 2015 TurkDeck: Physical Virtual Reality Based on People. In Proceedings of the 28th Annual ACM Symposium on User Interface Software \& Technology (UIST '15). ACM New York, NY, USA, 417-426. DOI : http://dx.doi.org/10.1145/2807442.2807463

[9] Inrak Choi, Eyal Ofek, Hrvoje Benko, Mike Sinclair, and Christian Holz. 2018. CLAW: A Multifunctional Handheld Haptic Controller for Grasping, Touching, and Triggering in Virtual Reality. In Proceedings of the 2018 CHI Conference on Human Factors in Computing Systems (CHI '18). ACM, New York, NY, USA, Article 654, 13 pages. DOI: http://dx.doi.org/10.1145/3173574.3174228

[10] Lauri Connelly, Yicheng Jia, Maria L. Toro, Mary Ellen Stoykov, Robert V. Kenyon, and Derek G. Kamper. 2010. A Pneumatic Glove and Immersive Virtual Reality Environment for Hand Rehabilitative Training After Stroke. IEEE Transactions on Neural Systems and Rehabilitation Engineering 18, 5 (Oct 2010), 551-559. DOI : http://dx.doi.org/10.1109/TNSRE.2010.2047588

[11] Fabien Danieau, Philippe Guillotel, Olivier Dumas, Thomas Lopez, Bertrand Leroy, and Nicolas Mollet. 2018. HFX Studio: Haptic Editor for Full-body Immer sive Experiences. In Proceedings of the 24th ACM Symposium on Virtual Reality Software and Technology (VRST '18). ACM, New York, NY, USA, Article 37, 9 pages. DOI : http://dx.doi.org/10.1145/3281505.3281518

[12] Tim Duente, Max Pfeiffer, and Michael Rohs. 2017. Zap++: A 20-channel Electrical Muscle Stimulation System for Fine-grained Wearable Force Feedback. In Proceedings of the 19th International Conference on Human-Computer Interaction with Mobile Devices and Services (MobileHCI '17). ACM, New York, NY, USA Article 1, 13 pages. DOI : http://dx.doi.org/10.1145/3098279.3098546

[13] Ayaka Ebisu, Satoshi Hashizume, Kenta Suzuki, Akira Ishii, Mose Sakashita and Yoichi Ochiai. 2017. Stimulated Percussions: Method to Control Human for Learning Music by Using Electrical Muscle Stimulation. In Proceedings of the 8th Augmented Human International Conference (AH '17). ACM, New York, NY, USA Article 33, 5 pages. DOI : http://dx.doi.org/10.1145/3041164.3041202

[14] Farzam Farbiz, Zhou Hao Yu, Corey Manders, and Waqas Ahmad. 2007. An Electrical Muscle Stimulation Haptic Feedback for Mixed Reality Tennis Game. In ACM SIGGRAPH 2007 Posters (SIGGRAPH '07). ACM, New York, NY, USA, Article 140. DOI : http://dx.doi.org/10.1145/1280720.1280873

[15] Bryan E. Feldman, James F. O’Brien, and Okan Arikan. 2003. Animating Suspended Particle Explosions. In ACM SIGGRAPH 2003 Papers (SIGGRAPH '03). ACM New York, NY, USA, 708-715. DOI : http://dx.doi.org/10.1145/1201775.882336

[16] Takashi Goto, Benjamin Tag, Kai Kunze, and Tilman Dingler. 2018. Towards Enhancing Emotional Responses to Media Using Auto-Calibrating Electric Muscle Stimulation (EMS). In Proceedings of the 9th Augmented Human International Conference (AH '18). ACM, New York, NY, USA, Article 29, 2 pages. DOI : http: //dx.doi.org/10.1145/3174910.3174939

[17] Kris Graft. 2009. Analysis: The Psychology Behind Item Collecting And Achievement Hoarding. https://www.gamasutra.com/view/news/114668/Analysis The Psychology_Behind_Item_Collecting_And_Achievement_Hoarding.php. (2009). Accessed 10 September 2018.

[18] Xiaochi Gu, Yifei Zhang, Weize Sun, Yuanzhe Bian, Dao Zhou, and Per Ola Kristensson. 2016. Dexmo: An Inexpensive and Lightweight Mechanical Exoskeleton for Motion Capture and Force Feedback in VR. In Proceedings of the $2016 \mathrm{CHI}$ Conference on Human Factors in Computing Systems (CHI '16). ACM, New York, NY, USA, 1991-1995. DOI : http://dx.doi.org/10.1145/2858036.2858487

[19] Sidhant Gupta, Dan Morris, Shwetak N. Patel, and Desney Tan. 2013. AirWave: Non-contact Haptic Feedback Using Air Vortex Rings. In Proceedings of the 2013 ACM International foint Conference on Pervasive and Ubiquitous Computing (UbiComp '13). ACM, New York, NY, USA, 419-428. DOI : http://dx.doi.org/10. $1145 / 2493432.2493463$

[20] Ping-Hsuan Han, Yang-Sheng Chen, Kong-Chang Lee, Hao-Cheng Wang, ChiaoEn Hsieh, Jui-Chun Hsiao, Chien-Hsing Chou, and Yi-Ping Hung. 2018. Haptic Around: Multiple Tactile Sensations for Immersive Environment and Interaction in Virtual Reality. In Proceedings of the 24th ACM Symposium on Virtual Reality Software and Technology (VRST '18). ACM, New York, NY, USA, Article 35, 10 pages. DOI : http://dx.doi.org/10.1145/3281505.3281507

[21] Hugh Hancock. 2002. Better Game Design Through Cutscenes. https://www. gamasutra.com/view/feature/131410/better_game_design_through_php. (2002) Accessed 10 September 2018

[22] Mahmoud Hassan, Florian Daiber, Frederik Wiehr, Felix Kosmalla, and Antonio
Krüger. 2017. FootStriker: An EMS-based Foot Strike Assistant for Running. Proc. ACM Interact. Mob. Wearable Ubiquitous Technol. 1, 1, Article 2 (March 2017), 18 pages. DOI : http://dx.doi.org/10.1145/3053332

[23] Mariam Hassib, Max Pfeiffer, Stefan Schneegass, Michael Rohs, and Florian Alt. 2017. Emotion Actuator: Embodied Emotional Feedback Through Electroencephalography and Electrical Muscle Stimulation. In Proceedings of the 2017 CHI Conference on Human Factors in Computing Systems (CHI '17). ACM, New York, NY, USA, 6133-6146. DOI : http://dx.doi.org/10.1145/3025453.3025953

[24] Christian Holz, Eyal Ofek, Mike Sinclair, Evan Strasnick, and Hrvoje Benko. 2018. Haptic Links: Bimanual Haptics for Virtual Reality Using Variable Stiffness Actuation, In CHI '18. (April 2018).

[25] Lode Hoste and Beat Signer. 2013. Water Ball Z: An Augmented Fighting Game Using Water As Tactile Feedback. In Proceedings of the 8th International Conference on Tangible, Embedded and Embodied Interaction (TEI '14). ACM, New York, NY, USA, 173-176. DOI : http://dx.doi.org/10.1145/2540930.2540946

[26] Ali Israr, Zachary Schwemler, John Mars, and Brian Krainer. 2016. VR360HD: A VR360\&Deg; Player with Enhanced Haptic Feedback. In Proceedings of the 22Nd ACM Conference on Virtual Reality Software and Technology (VRST '16). ACM, New York, NY, USA, 183-186. DOI : http://dx.doi.org/10.1145/2993369.2993404

[27] Shubham Jain, Shubham Sharma, and Dhawal Babbar. 2017. Star-Force: A Playful Implementation of the Jedi-force. In Proceedings of the Eleventh International Conference on Tangible, Embedded, and Embodied Interaction (TEI '17). ACM, New York, NY, USA, 761-766. DOI : http://dx.doi.org/10.1145/3024969.3041098

[28] Genichi Kawada and Takashi Kanai. 2011. Procedural Fluid Modeling of Explosion Phenomena Based on Physical Properties. In Proceedings of the 2011 ACM SIGGRAPH/Eurographics Symposium on Computer Animation (SCA '11). ACM, New York, NY, USA, 167-176. DOI : http://dx.doi.org/10.1145/2019406.2019429

[29] Jarrod Knibbe, Paul Strohmeier, Sebastian Boring, and Kasper Hornbæk. 2017. Automatic Calibration of High Density Electric Muscle Stimulation. Proc. ACM Interact. Mob. Wearable Ubiquitous Technol. 1, 3, Article 68 (Sept. 2017), 17 pages. DOI : http://dx.doi.org/10.1145/3130933

[30] Pascal Knierim, Thomas Kosch, Valentin Schwind, Markus Funk, Francisco Kiss, Stefan Schneegass, and Niels Henze. 2017. Tactile Drones - Providing Immersive Tactile Feedback in Virtual Reality Through Quadcopters. In Proceedings of the 2017 CHI Conference Extended Abstracts on Human Factors in Computing Systems (CHI EA '17). ACM, New York, NY, USA, 433-436. DOI : http://dx.doi.org/10.1145/ 3027063.3050426

[31] Michinari Kono, Yoshio Ishiguro, Takashi Miyaki, and Jun Rekimoto. 2018a. Design and Study of a Multi-Channel Electrical Muscle Stimulation Toolkit for Human Augmentation. In Proceedings of the 9th Augmented Human International Conference (AH '18). ACM, New York, NY, USA, Article 11, 8 pages. DOI : http: //dx.doi.org/10.1145/3174910.3174913

[32] Michinari Kono, Takashi Miyaki, and Jun Rekimoto. 2018b. In-pulse: Inducing Fear and Pain in Virtual Experiences. In Proceedings of the 24th ACM Symposium on Virtual Reality Software and Technology (VRST '18). ACM, New York, NY, USA, Article 40, 5 pages. DOI : http://dx.doi.org/10.1145/3281505.3281506

[33] Anna Kozlova. 2018. How do Teslasuit technologies change enterprise training? https://teslasuit.io/blog/haptic-feedback/how-teslasuit-changes-enterprisetraining. (September 2018). Accessed 10 September 2018

[34] Ernst Kruijff, Dieter Schmalstieg, and Steffi Beckhaus. 2006. Using Neuromuscular Electrical Stimulation for Pseudo-haptic Feedback. In Proceedings of the ACM Symposium on Virtual Reality Software and Technology (VRST '06). ACM, New York, NY, USA, 316-319. DOI : http://dx.doi.org/10.1145/1180495.1180558

[35] Lawrence Kutner and Cheryl Olson. 2008. Grand theft childhood: The surprising truth about violent video games and what parents can do. Simon and Schuster.

[36] Pedro Lopes and Patrick Baudisch. 2017. Immense Power in a Tiny Package: Wearables Based on Electrical Muscle Stimulation. IEEE Pervasive Computing 16, 3 (2017), 12-16. DOI : http://dx.doi.org/10.1109/MPRV.2017.2940953

[37] Pedro Lopes, Alexandra Ion, and Patrick Baudisch. 2015. Impacto: Simulating Physical Impact by Combining Tactile Stimulation with Electrical Muscle Stimulation. In Proceedings of the 28th Annual ACM Symposium on User Interface Software \& Technology (UIST '15). ACM, New York, NY, USA, 11-19. DOI: http://dx.doi.org/10.1145/2807442.2807443

[38] Pedro Lopes, Alexandra Ion, Sijing You, and Patrick Baudisch. 2018. Adding Force Feedback to Mixed Reality Experiences and Games using Electrical Muscle Stimulation. In Proceedings of the 2018 CHI Conference on Human Factors in Computing Systems (CHI '18). ACM, New York, NY, USA.

[39] Pedro Lopes, Patrik Jonell, and Patrick Baudisch. 2015. Affordance++: Allowing Objects to Communicate Dynamic Use. In Proceedings of the 33rd Annual ACM Conference on Human Factors in Computing Systems (CHI '15). ACM, New York, NY, USA, 2515-2524. DOI : http://dx.doi.org/10.1145/2702123.2702128

[40] Pedro Lopes, Sijing You, Lung-Pan Cheng, Sebastian Marwecki, and Patrick Baudisch. 2017. Providing Haptics to Walls \& Heavy Objects in Virtual Reality by Means of Electrical Muscle Stimulation. In Proceedings of the 2017 CHI Conference on Human Factors in Computing Systems (CHI '17). ACM, New York, NY, USA, 1471-1482. DOI : http://dx.doi.org/10.1145/3025453.3025600

[41] Ryohei Nagao, Keigo Matsumoto, Takuji Narumi, Tomohiro Tanikawa, and Michitaka Hirose. 2017. Infinite Stairs: Simulating Stairs in Virtual Reality 
Based on Visuo-haptic Interaction. In ACM SIGGRAPH 2017 Emerging Technologies (SIGGRAPH '17). ACM, New York, NY, USA, Article 14, 2 pages. DOI: http://dx.doi.org/10.1145/3084822.3084838

[42] Yoichi Nagashima. 2003. Bio-sensing Systems and Bio-feedback Systems for Interactive Media Arts. In Proceedings of the 2003 Conference on New Interfaces for Musical Expression (NIME '03). National University of Singapore, Singapore, Singapore, 48-53. http://dl.acm.org/citation.cfm?id=1085714.1085726

[43] Arinobu Niijima and Takefumi Ogawa. 2016. Virtual Food Texture by Electrical Muscle Stimulation. In Proceedings of the 2016 ACM International Symposium on Wearable Computers (ISWC '16). ACM, New York, NY, USA, 48-49. DOI http://dx.doi.org/10.1145/2971763.2971792

[44] Max Pfeiffer, Tim Duente, and Michael Rohs. 2016. Let Your Body Move: A Prototyping Toolkit for Wearable Force Feedback with Electrical Muscle Stimulation. In Proceedings of the 18th International Conference on Human-Computer Interaction with Mobile Devices and Services (MobileHCI '16). ACM, New York, NY, USA, 418-427. DOI : http://dx.doi.org/10.1145/2935334.2935348

[45] Max Pfeiffer, Tim Dünte, Stefan Schneegass, Florian Alt, and Michael Rohs. 2015 Cruise Control for Pedestrians: Controlling Walking Direction Using Electrical Muscle Stimulation. In Proceedings of the 33rd Annual ACM Conference on Human Factors in Computing Systems (CHI '15). ACM, New York, NY, USA, 2505-2514. DOI : http://dx.doi.org/10.1145/2702123.2702190

[46] Max Pfeiffer, Stefan Schneegass, Florian Alt, and Michael Rohs. 2014. Let Me Grab This: A Comparison of EMS and Vibration for Haptic Feedback in Free-hand Interaction. In Proceedings of the 5th Augmented Human International Conference (AH '14). ACM, New York, NY, USA, Article 48, 8 pages. DOI : http://dx.doi.org/ $10.1145 / 2582051.2582099$

[47] Daniel Roth, Kristoffer Waldow, Felix Stetter, Gary Bente, Marc Erich Latoschik, and Arnulph Fuhrmann. 2016. SIAMC: A Socially Immersive Avatar Mediated Communication Platform. In Proceedings of the 22Nd ACM Conference on Virtual Reality Software and Technology (VRST '16). ACM, New York, NY, USA, 357-358. DOI : http://dx.doi.org/10.1145/2993369.2996302

[48] Dominik Schmidt, Rob Kovacs, Vikram Mehta, Udayan Umapathi, Sven Köhler, Lung-Pan Cheng, and Patrick Baudisch. 2015. Level-Ups: Motorized Stilts That Simulate Stair Steps in Virtual Reality. In Proceedings of the 33rd Annual ACM Conference on Human Factors in Computing Systems (CHI '15). ACM, New York, NY, USA, 2157-2160. DOI : http://dx.doi.org/10.1145/2702123.2702253

[49] Dominik Schmidt, Raf Ramakers, Esben W. Pedersen, Johannes Jasper, Sven
Köhler, Aileen Pohl, Hannes Rantzsch, Andreas Rau, Patrick Schmidt, Christoph Sterz, Yanina Yurchenko, and Patrick Baudisch. 2014. Kickables: Tangibles for Feet. In Proceedings of the SIGCHI Conference on Human Factors in Computing Systems (CHI '14). ACM, New York, NY, USA, 3143-3152. DOI : http://dx.doi.org/ $10.1145 / 2556288.2557016$

[50] Samuel B. Schorr and Allison M. Okamura. 2017. Fingertip Tactile Devices for Virtual Object Manipulation and Exploration. In Proceedings of the $2017 \mathrm{CHI}$ Conference on Human Factors in Computing Systems (CHI '17). ACM, New York, NY, USA, 3115-3119. DOI : http://dx.doi.org/10.1145/3025453.3025744

[51] Thomas Schubert, Frank Friedmann, and Holger Regenbrecht. 2001. The Experience of Presence: Factor Analytic Insights. Presence: Teleoperators and Virtual Environments 10, 3 (2001), 266-281. DOI: http://dx.doi.org/10.1162/ 105474601300343603

[52] Andrew Selle, Nick Rasmussen, and Ronald Fedkiw. 2005. A Vortex Particle Method for Smoke, Water and Explosions. ACM Trans. Graph. 24, 3 (July 2005), 910-914. DOI : http://dx.doi.org/10.1145/1073204.1073282

[53] Rajinder Sodhi, Ivan Poupyrev, Matthew Glisson, and Ali Israr. 2013. AIREAL: Interactive Tactile Experiences in Free Air. ACM Trans. Graph. 32, 4, Article 134 (July 2013), 10 pages. DOI : http://dx.doi.org/10.1145/2461912.2462007

[54] Emi Tamaki, Terence Chan, and Ken Iwasaki. 2016. UnlimitedHand: Input and Output Hand Gestures with Less Calibration Time. In Proceedings of the 29th Annual Symposium on User Interface Software and Technology (UIST '16 Adjunct). ACM, New York, NY, USA, 163-165. DOI : http://dx.doi.org/10.1145/ 2984751.2985743

[55] Emi Tamaki, Takashi Miyaki, and Jun Rekimoto. 2011. PossessedHand: Techniques for Controlling Human Hands Using Electrical Muscles Stimuli. In Proceedings of the SIGCHI Conference on Human Factors in Computing Systems (CHI '11). ACM, New York, NY, USA, 543-552. DOI : http://dx.doi.org/10.1145/1978942. 1979018

[56] TENS-EMS. 2018. TENS EMS Combo Device. https://tens-ems.com/en/tens-andems-devices/tens-ems-combo-device. (2018). Accessed 10 September 2018.

[57] Gary D. Yngve, James F. O’Brien, and Jessica K. Hodgins. 2000. Animating Explosions. In Proceedings of the 27th Annual Conference on Computer Graphics and Interactive Techniques (SIGGRAPH '00). ACM Press/Addison-Wesley Publishing Co., New York, NY, USA, 29-36. DOI : http://dx.doi.org/10.1145/344779.344801

[58] Vladimir M Zatsiorsky and William J Kraemer. 2006. Science and practice of strength training. Human Kinetics. 